\title{
Editorial
}

\section{Data-Driven Urban Mobility Modeling and Analysis}

\author{
Xiaolei Ma, ${ }^{1}$ Guohui Zhang, ${ }^{2}$ and Xiaoyue Liu ${ }^{3}$ \\ ${ }^{1}$ School of Transportation Science and Engineering, Beihang University, Beijing 100191, China \\ ${ }^{2}$ Department of Civil \& Environment Engineering, University of Hawaii, Manoa, HI, USA \\ ${ }^{3}$ Department of Civil \& Environment Engineering, University of Utah, Salt Lake City, UT, USA \\ Correspondence should be addressed to Xiaolei Ma; xiaolei@buaa.edu.cn
}

Received 14 June 2017; Accepted 14 June 2017; Published 30 July 2017

Copyright (C) 2017 Xiaolei Ma et al. This is an open access article distributed under the Creative Commons Attribution License, which permits unrestricted use, distribution, and reproduction in any medium, provided the original work is properly cited.

With increasing economic and social activities, travel demand has increased significantly over the past several decades, overloading many already congested roadways. The widening gap between travel demand and infrastructure supply has worsened the levels of congestion worldwide, resulting in many urban mobility, safety, and environmental issues, such as severe congestion, lengthened travel time, increased risk of traffic accidents, excessive fuel consumption, increased air pollution, and significant public health issues. The concept of smart cities has been gaining popularity, which is to leverage big data analytics, sensing technologies, and Internet of Things (IoT) to move people and goods faster, cheaper, and more efficiently. As heterogeneous data and computational resources become available, the development of data-driven approaches has been advancing as well for modeling and analyzing urban mobility. This special issue serves as a major platform to facilitate the discussion and exchange of research ideas and technology development, encourage multidimensional knowledge sharing, and enhance research activities in data-driven urban mobility modeling and analysis. In total, seven papers are included in this special issue and are summarized as follows.

There are several articles focusing on developing datadriven approaches to improve public transit system efficiency and accessibility. J. Ma et al. proposed a methodological framework to address the issue of customized bus network design based on large-scale travel demand data. A route selection model considering operation cost and social welfare was built, followed by a branch-and-bound-based solution method for model solving. An empirical study in Beijing, China, validated the effectiveness of the proposed framework.
Z. Cai et al. proposed a Trip Coverage Index (TCI) based on mobile phone data to assess transit accessibility. TCI considered both the individual-level transit trip coverage and spatial distribution and was then applied to a transit network in Hangzhou, China.

From the perspective of car-based traffic operation and management, several articles talk about utilizing sensing technology and simulation data to analyze drivers' behaviors, route choices, or road traffic network structure. D. $\mathrm{Xu}$ et al. developed a compression method based on LZW encoding and principle component analysis. Six typical road segments in Beijing were tested using the proposed method and presented a high reconstruction accuracy. $H$. Chen et al. clustered drivers' travel characteristics based on license plate recognition data in Shenzhen, China. Each traveler's spatiotemporal variability and activity pattern are taken into account, resulting in six groups in weekdays and three groups in weekends. J. Wang et al. proposed a route choice prediction model in the context of connected vehicles. Five characteristics indexes including compliance rate, following rate, penetration rate, release delay time, and congestion level were built. A simulation scenario demonstrated the effectiveness of the proposed model with the average root mean square error as $3.19 \%$. Y. Lu et al. introduced a novel improved stochastic Gompertz diffusion process to explain the relationship between vehicle ownership and GDP percapita. Based on the data from US, UK, Japan, and Korea from 1960 to 2008, the proposed model performed well in the fitting process and predicted that China is still on the initial stage of motorization. 
The remaining two articles investigated travelers' psychologies and behaviors using survey data. C. Ding et al. examined the impact of attitudes to walking and cycling on commute mode choice and used survey data to establish an integrated discrete choice model and structural equation model. A comparison confirmed that the proposed hybrid model outperforms other traditional models. F. Pan et al. explored the influence of vehicular countdown signals on driving psychologies and behaviors. An online survey with 1051 valid questionnaires was undertaken and analyzed. Results showed that most drivers prefer countdown signal controls and female drivers are more conservative before the green countdown ends.

Xiaolei Ma Guohui Zhang Xiaoyue Liu 


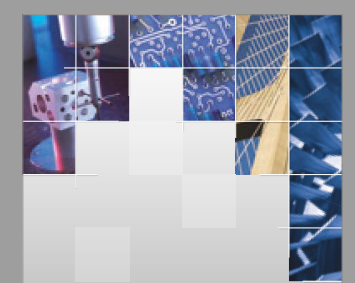

\section{Enfincering}
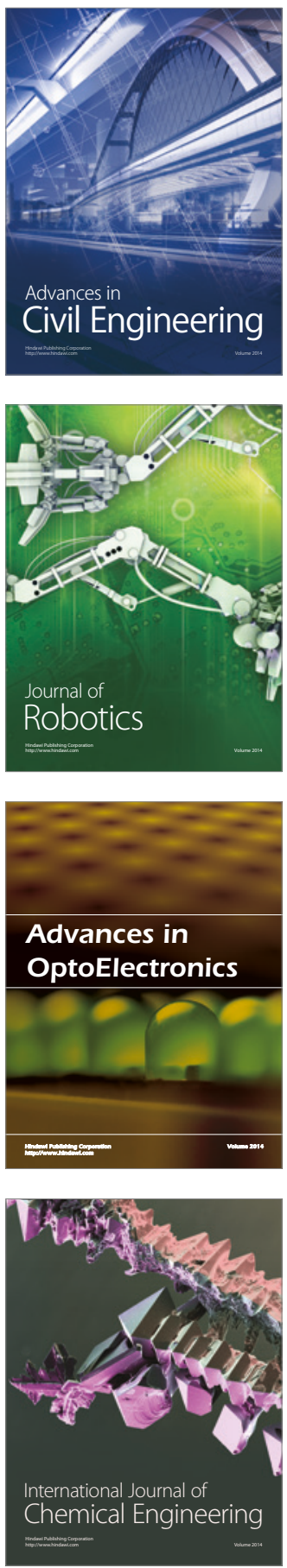

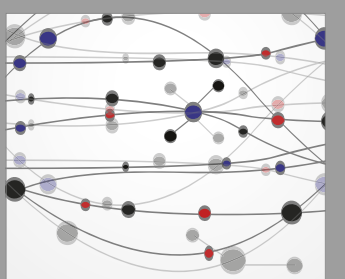

The Scientific World Journal

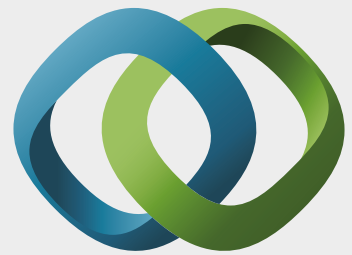

\section{Hindawi}

Submit your manuscripts at

https://www.hindawi.com
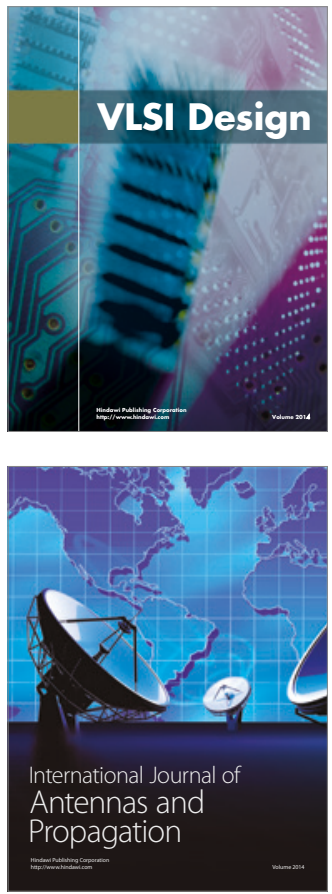

\section{Rotating}

Machinery
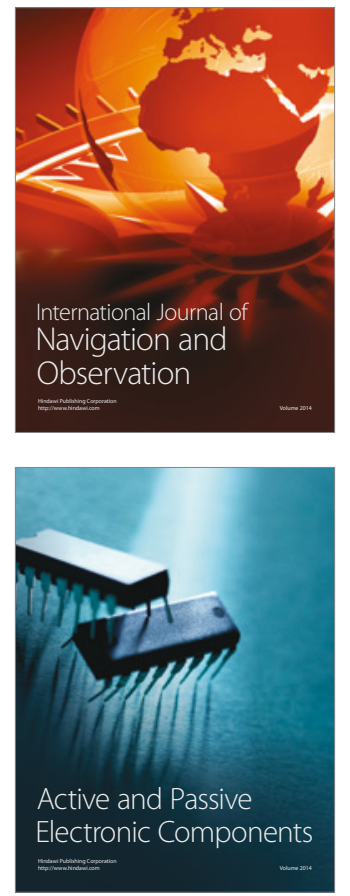
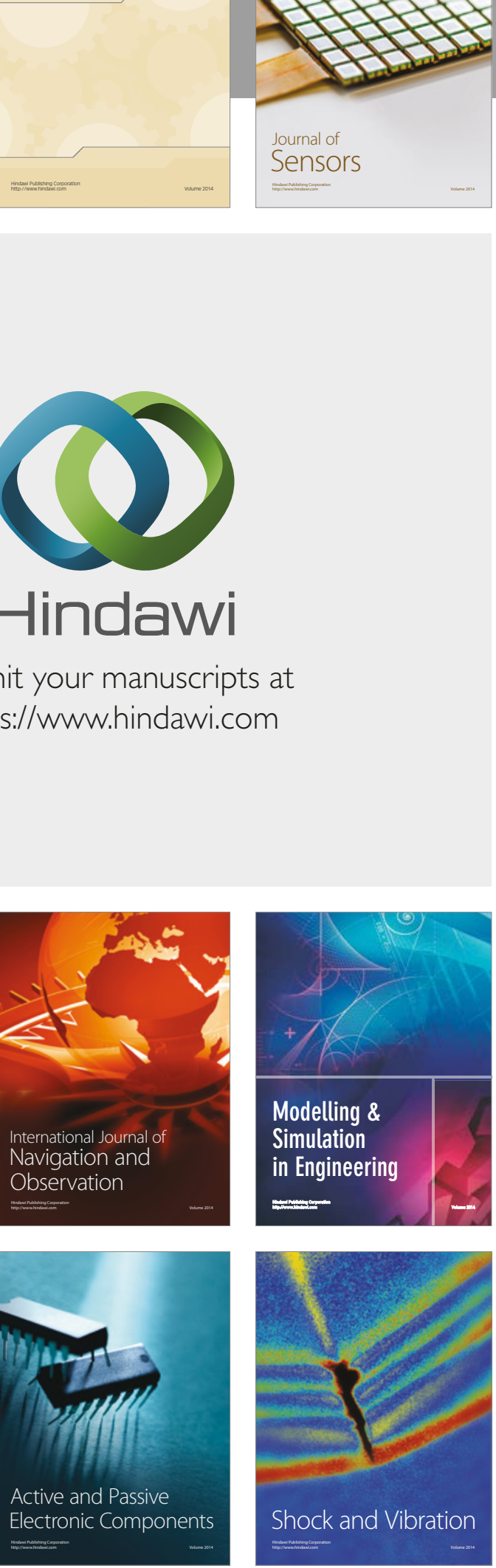
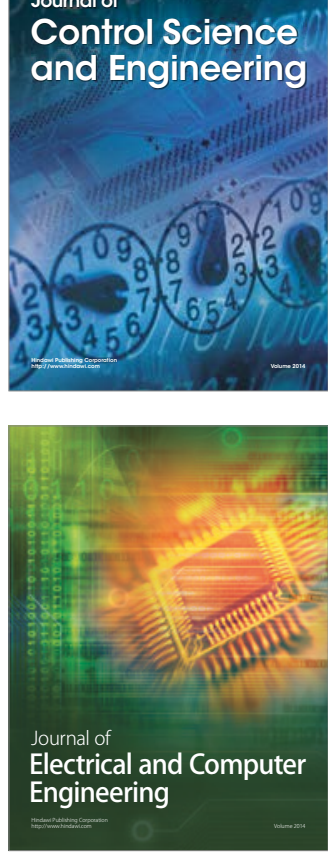

Distributed

Journal of

Control Science

and Engineering
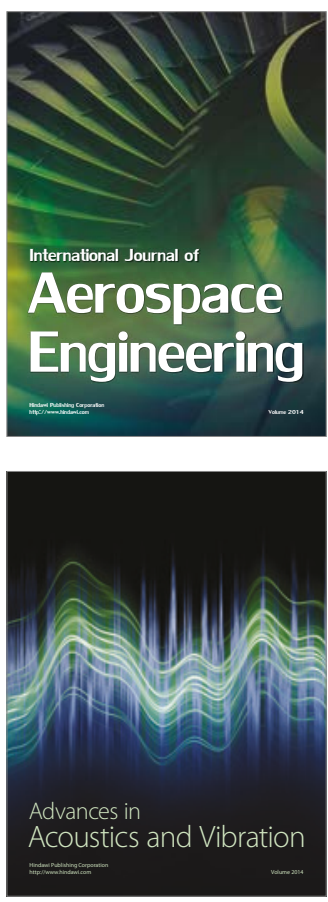

Sensor Networks 